\title{
Antibiotic Susceptibility Profiling of Gram-Negative Bacteria Causing Upper Respiratory Tract Infections in Hyderabad, Sindh
}

\author{
Ghulam A. Maka, Samreen Shah, Shaista Bano, Sarfraz A. Tunio* \\ Institute of Microbiology, University of Sindh, Jamshoro, Pakistan (gasgharmaka@gmail.com, sshah.mb@gmail.com, \\ shaista.bano@usindh.edu.pk,sarfraz.tunio@usindh.edu.pk) \\ *Correspondence: sarfraz.tunio@usindh.edu.pk
}

\begin{abstract}
Respiratory tract infections (RTIs) are important clinical problems and among the commonest infectious diseases throughout the world. Several factors including gender, age and season have been shown to influence the prevalence rates of RTIs. The current study aimed to isolate and identify bacteria causing of upper respiratory tract (URT) infections and to determine the antibiotic susceptibility patterns of the isolated bacteria. A total of 201 sputum and swab samples were collected from patients from August 2015 to March 2016 and investigated for Gram-negative pathogenic bacteria. The antibiotic sensitivity of isolated bacteria was performed using Kirby Bauer Disc diffusion method. Isolation and identification of the bacteria were carried out using conventional methods including microscopic, cultural and biochemical testing. Out of 201 samples, 29.85\% $(n=60)$ yielded bacterial growth in which $20.9 \%(n=42)$ belonged to male while $8.96 \%(\mathrm{n}=18)$ were from female patients. Among the isolates, Pseudomonas aeruginosa was the most frequent bacteria accounting $48.33 \%(n=29)$, followed by Klebsiella pneumoniae $45 \%(n=27)$ and $E$. coli $6.67 \%$ ( $n=04)$. The data of antibiotic susceptibility profiling demonstrated that Cefoperazone sulbactam, Meropenem, Piperacillin Tazobactam and Amikacin were highly effective against all isolated bacteria. However, Ampicillin, Cephradine, Ofloxacin and Co-trimoxazole were found the least effective antibiotics against all isolated bacteria. In summary, an increasing trend in the resistance against antibiotics which are more frequently prescribed, such as Cephradine, Ampicillin and Co-trimoxazole was observed. Therefore, a continuous surveillance of antibiotic resistance trends of pathogens is needed to ensure appropriate recommendations for the treatment of the URTIs.
\end{abstract}

Keywords: Upper Respiratory Tract, Antibiotic Resistance, Gram-Negative Bacteria, Klebsiella pneumonia, Pseudomonas aeruginosa, E. coli

Received: March 05, 2020 / Accepted: April 22, 2020 / Online: April 23, 2020

\section{INTRODUCTION}

Respiratory tract infection (RTI) can be defined as any infectious disease of the upper respiratory tract (URT) such as laryngitis, the common cold, pharyngitis, acute rhinitis, and acute otitis media. Also, the lower respiratory tract including bronchiolitis, acute bronchitis, tracheitis and pneumonia. Children and adults are frequently reported to suffer from RTI worldwide with higher rates of mortality and morbidity in developing countries (Kunwor et al., 2014). An estimated 50 million deaths have been reported to occur every year globally (El-Mahmood et al., 2010). The infections are commonly treated with antibiotics in children and adults in primary care. The antibiotic resistance patterns and the prevalence of RTI have been shown to vary based on several factors including age, gender, locality and season (Siddalingappa et al., 2013). Respiratory tract plays a crucial role in breathing processes as being a vital part of the body system (Taura et al., 2013). RTI is very common due to the direct contact of the respiratory tract with the physical environment comprising various airborne pathogens (ElMahmood et al., 2010). The respiratory system consists of the upper respiratory tract and lower respiratory tracts (Taura et al., 2013). URT can be infected by the invasion of the mucosa lining the upper airway (El-Mahmood et al., 2010). Pathogens enter the URT through inhalation, where they may colonize the mucosa and mostly remain in the asymptomatic phase. Pathogenic bacteria may reach the middle ear via nasopharynx and cause acute otitis media, or may further disseminate to lung parenchyma and cause lower respiratory tract infections (Xirogianni et al., 2013). The majority of RTIs involve URT while only $5 \%$ have been reported to cause lower respiratory tract infections (Siddalingappa et al., 2013).

Bacteria commonly causing RTIs include both gramnegative and gram-positive bacteria such as Pseudomonas spp., Haemophilus influenzae Proteus spp., Klebsiella spp., Streptococcus spp., Staphylococcus spp., Enterobacter spp., Acinetobacter spp., and (Manikandan and Amsath, 2013). The use of antibiotics in the treatment of URT infections is another area of serious concern as URTs are usually selflimiting and complications may reduce if antibiotics are withheld. 
Thus the irrational and misuse of antibiotics have led to a rise in cases of bacterial resistance and many important antibiotics are slowly and steadily losing their efficacy, threatening to push us back in pre-antibiotic era (Sharma and Agrawal, 2016). Moreover, RTIs have a significant economic impact as the patients may be less productive and the prescription of costly antibiotics is a huge economical burden despite the infections is not caused by bacteria. In Pakistan, due to either expensive laboratory tests or unavailability of facilities of modern diagnostic procedures in laboratories, the RTIs are mostly treated empirically. Thus, continuous surveillance studies are an important tool in identifying the local antimicrobial resistance patterns of indigenous pathogens to assist the clinicians in empirical therapy (Zafar et al., 2008). Thus, the present study was designed to investigate the bacteriological profile with an emphasis on Gram-negative bacteria and their susceptibility patterns to different commonly used antibiotics.

\section{MATERIALS AND METHODS}

\section{A. Study Site, Sampling and Duration}

This cross-sectional study was conducted at the Institute of Microbiology, University of Sindh during August 2015 to March 2016. A total of 201 samples (sputum and throat swabs) were collected aseptically from the patients attending the diagnostic and research laboratory, Hyderabad. The sputum samples were collected into sterile, well-labeled wide mouthed screw capped plastic bottles. Whereas the throat swab samples were collected using a sterile cotton swab.

\section{B. Sample Processing}

All samples were processed immediately after collection. Initially, the samples were plated out on Blood agar and MacConkey's agar. The inoculated plates were incubated at $37^{\circ} \mathrm{C}$ for 24 hours. The following day, discrete colonies were selected and grown in pure culture for further identification and antimicrobial susceptibility testing.

\section{Identification of Bacterial Isolates of URT}

Isolation and identification of all isolated bacteria was carried as described previously (Bano et al., 2015). Briefly, all bacteria were identified using conventional methods including Gram-staining, morphological characteristics (shape, size and arrangement), cultural characteristics on diagnostic media and biochemical tests such as Urease test, Simmons' citrate test, Sulphide Indole Motility (SIM) test, and Oxidase test and Triple sugar iron agar (TSI) test.

\section{Antibiotic Sensitivity Profiling}

The antibacterial susceptibility testing was performed using the Kirby-Bauer disk diffusion method as described previously (Bano et al., 2014). Briefly, with the help of a sterile cotton swab, a lawn of overnight bacterial culture was prepared onto Mueller-Hinton agar plates (Oxoid). The plates were allowed to dry and then appropriate antibiotic discs were gently placed on the agar plates and slightly pressed with to ensure its contact with agar plates. The plates were then incubated at $37^{\circ} \mathrm{C}$ for 24 hours. The standard antibiotic discs were used included, Amoxicillin/

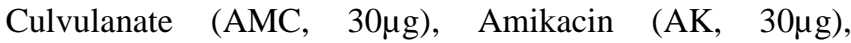

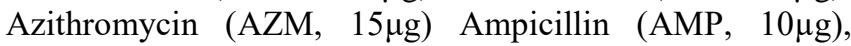

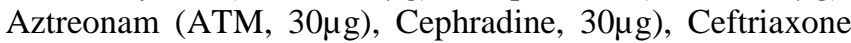
$(\mathrm{CRO}, 30 \mu \mathrm{g})$, Ceftazidime (CAZ, 30 $\mathrm{g})$. Cefoperazone

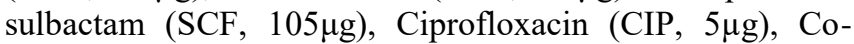
Trimoxazole (SXT, 1.25/23.75 $\mu \mathrm{g}$ ), Levofloxacin (LEV,

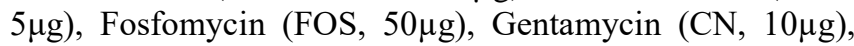

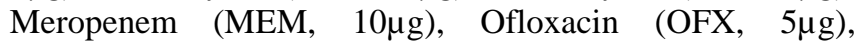

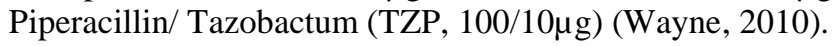

\section{RESULTS}

Out of 201 samples investigated for bacteriological analysis, $29.85 \%(n=60)$ yielded bacterial growth of a single bacteria, which consisted of $20.90 \%$ males and $8.96 \%$ females. Bacterial profiling showed that all positive samples had only Gram-negative bacteria (Figure 1). Among the positive samples, majority was from males patients.

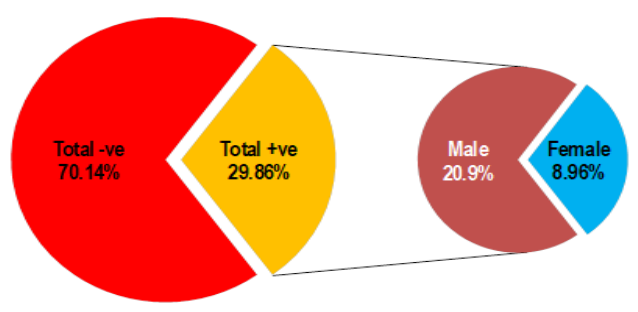

Figure 1. Pie chart showing gender wise distribution of total samples processed in this study.

\section{A. Age and Gender-Wise Distribution of URT}

Samples were collected from the age range of 4 to 90 years old individuals. The respiratory tract infections were more common in males than females in all age groups except the age group of 1-10 which has an equal number of patients. The age group of 31-40 years yielded the highest number of positivity for bacterial cultures (Figure 2). The age group of 1-10 years age, however, showed the lowest positivity for respiratory pathogens.

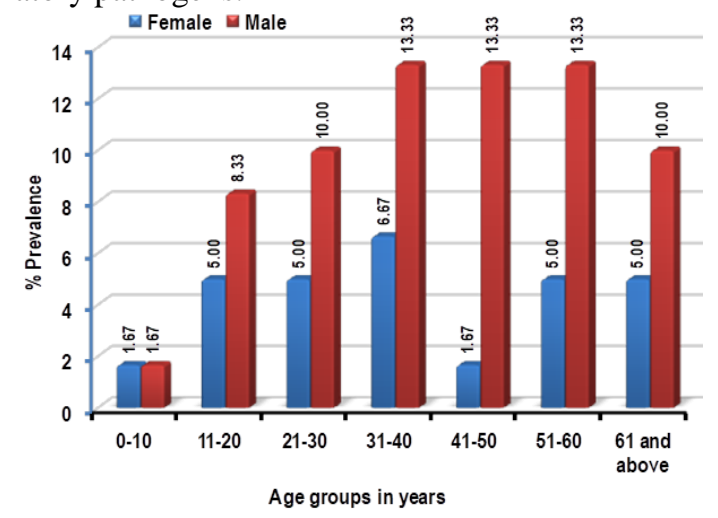

Figure 2. Age and gender-wise distribution of positive samples. 


\section{B. Distribution of Bacterial Isolates of URT}

In the present study, 60 bacterial isolates belonging to the Gram-negative group only were recovered from sputum and swab samples of URT. Among them, the most frequent isolate was Pseudomonas aeruginosa which accounted $48.33 \%(\mathrm{n}=29)$ of the isolates followed by Klebsiella pneumoniae $45 \%(\mathrm{n}=27)$, and E. coli $6.67 \%(\mathrm{n}=4)$. The majority of samples belonged to male patients and the age group 31-40 years was found to be favorable to the infection in the present study.

\section{Antibiotic Sensitivity Patterns of URT Bacterial Isolates}

In vitro antibiogram of all isolates was performed using various commonly used antibiotics (Figure 3 ). The findings demonstrated that Cefoperazone sulbactam, Meropenem, Piperacillin Tazobactam and Amikacin were effective for upper respiratory tract bacterial isolates whereas the bacteria showed resistance against Cephradine, Ampicillin, Ofloxacin and Co-Trimoxazole. P. aeruginosa showed the highest sensitivity to Piperacillin Tazobactam (90\%), Cefoperazone Sulbactam (88.24\%) and Amikacin (85.71\%). Cephradine, Ampicillin and Augmentin and co-trimoxazole were least effective. Klebsiella pneumoniae were found highly sensitive to Cefoperazone Sulbactam (100\%) and Meropenem (92\%) whereas Ampicillin was the least effective against them. Meropenem, Fosfomycin, Amikacin, Piperacillin Tazobactam, Cefoperazone Sulbactam showed the highest efficacy $(100 \%)$ against E. coli. However, Ampicillin, Ceftriaxone and Co-trimoxazole, Levofloxacin, Ofloxacin, were less effective against E. coli (Table 1).

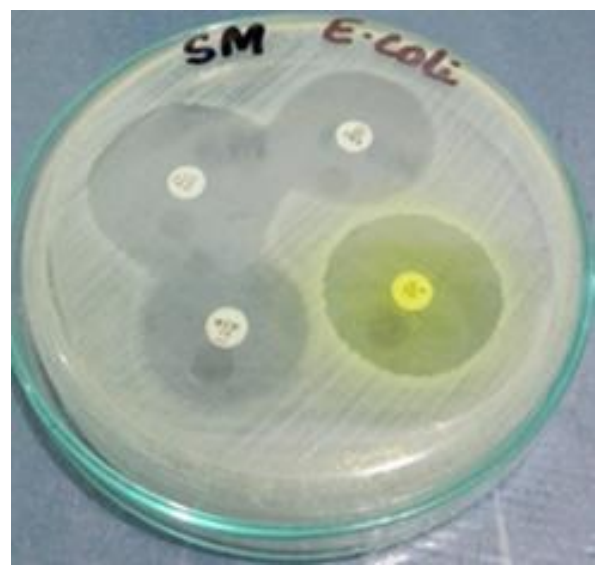

Figure 3. Muller Hinton agar plate showing a representative result of the antibiotic sensitivity testing using the disc diffusion method

\section{DISCUSSION}

The present study reports the identification of the bacterial agents' causing URTIs and their antibiotic susceptibility patterns at Hyderabad, Sindh. URTIs are usually of viral origin and may resolve spontaneously, however, if the symptoms persist for 10-14 days then antibiotic treatment is needed.
Table 1. Antibiotic sensitivity patterns of Gram-negative URT bacterial isolates, $\mathrm{S}=$ sensitive, $\mathrm{I}=$ intermediate, and $\mathrm{R}=$ resistant.

\begin{tabular}{|c|c|c|c|c|c|c|c|c|c|}
\hline Organisms & \multicolumn{3}{|c|}{$\begin{array}{c}\text { P. aeruginosa } \\
(\mathrm{n}=29)\end{array}$} & \multicolumn{3}{c|}{$\begin{array}{c}\text { K. pneumonia } \\
(\mathrm{n}=27)\end{array}$} & \multicolumn{3}{c|}{$\begin{array}{c}\text { E. coli } \\
(\mathrm{n}=04)\end{array}$} \\
\hline Antibiotics & $\mathrm{S} \%$ & $\mathrm{I} \%$ & $\mathrm{R} \%$ & $\mathrm{~S} \%$ & $\mathrm{I} \%$ & $\mathrm{R} \%$ & $\mathrm{~S} \%$ & $\mathrm{I} \%$ & $\mathrm{R} \%$ \\
\hline Amoxicillin & 13.33 & 3.33 & 83.33 & 66.67 & 11.11 & 22.22 & 75 & 0 & 25 \\
\hline Amikacin & 85.71 & 3.57 & 10.71 & 69.57 & 0 & 30.43 & 100 & 0 & 0 \\
\hline Azithromycin & 59.26 & 3.70 & 37.04 & 32 & 20 & 48.00 & 0 & 0 & 100 \\
\hline Ampicillin & 8.70 & 0 & 91.30 & 4.55 & 0 & 95.45 & 0 & 0 & 100 \\
\hline Aztreonam & 62.50 & 0 & 37.50 & 59.09 & 22.73 & 18.18 & 0 & 0 & 100 \\
\hline Cephradine & 5.56 & 0 & 94.44 & 17.65 & 0 & 82.35 & 0 & 0 & 100 \\
\hline Ceftriaxone & 63.64 & 0 & 36.36 & 27.27 & 9.09 & 63.64 & 0 & 0 & 100 \\
\hline Ceftazidime & 81.48 & 0 & 18.52 & 55.56 & 5.56 & 38.89 & 50 & 0 & 50 \\
\hline $\begin{array}{c}\text { Cefoperazone } \\
\text { / sulbactam }\end{array}$ & 88.24 & 0 & 11.76 & 100 & 0 & 0 & 100 & 0 & 0 \\
\hline Ciprofloxacin & 76 & 4 & 20 & 52.17 & 0 & 47.83 & 0 & 0 & 100 \\
\hline Co-trimoxazole & 17.39 & 0 & 82.61 & 34.78 & 0 & 65.22 & 0 & 0 & 100 \\
\hline Levofloxacin & 57.14 & 9.52 & 33.33 & 37.50 & 8.33 & 54.17 & 0 & 0 & 100 \\
\hline Fosfomycin & 29.17 & 0 & 70.83 & 62.50 & 0 & 37.50 & 100 & 0 & 0 \\
\hline Gentamycin & 71.43 & 0 & 28.57 & 66.67 & 7.41 & 25.93 & 25 & 25 & 50 \\
\hline Meropenem & 75.86 & 0 & 24.14 & 92 & 4 & 4 & 100 & 0 & 0 \\
\hline Ofloxacin & 63.33 & 6.67 & 30 & 37.50 & 0 & 62.50 & 0 & 0 & 100 \\
\hline $\begin{array}{l}\text { Piperacillin } \\
\text { tazobactam }\end{array}$ & 90 & 6.67 & 3.33 & 88.89 & 7.41 & 3.70 & 75 & 0 & 25 \\
\hline & & & & & & & & 50 \\
\hline
\end{tabular}

The current study demonstrated that URTIs were caused by three common Gram-negative bacteria which were frequently recovered from URTIs samples. Among the isolated bacteria, the $P$. aeruginosa was the most prevalent pathogen followed by $K$. pneumonia and then E. coli. Gender-wise distribution of the patients showed that the males were higher in number than females while the agewise distribution revealed that age group consisting 31-40 had the highest number of infected patients as compared to the other age groups such as younger or older age. The increased susceptibility of male patients may possibly be associated with the frequent exposure of the male patients to the polluted environment during their work.

The antibiotic sensitivity data of the isolates demonstrated that Piperacillin Tazobactam and Amikacin were highly effective against the isolated bacteria whereas Cephradine, Ampicillin, Ofloxacin and Co-Trimoxazole were least effective antibiotics. P. aeruginosa demonstrated highest sensitivity to Piperacillin Tazobactam followed by Cefoperazone Sulbactam and Amikacin whereas Cephradine, Ampicillin and Augmentin and co-trimoxazole were less effective antibiotics. Carbapenems are frequently used as a last choice in treating serious infections caused by Gram-negative bacteria. However, in our study, $24.14 \%$ of $P$. aeruginosa were resistant to Meropenem. The increasing 
resistance in $P$. aeruginosa against meropenem has also been reported by other studies (Rodríguez et al., 2009).

$K$. pneumoniae were highly sensitive to Cefoperazone Sulbactam followed by Meropenem whereas Ampicillin was the least effective antibiotic. E. coli were resistant to majority of antibiotics used in this study including Azithromycin, Ampicillin, Aztreonam, Cephradine, Ceftriaxone, Ciprofloxacin, Co-trimoxazole, Levofloxacin while sensitive to Amikacin, Fosfomycin, Cefoperazone / sulbactam and, Meropenem.

\section{CONCLUSIONS}

The data of antibiotic susceptibility testing revealed that Cefoperazone sulbactam, Meropenem, Piperacillin Tazobactam and Amikacin were more effective antibiotics against Gram-negative bacteria causing upper respiratory tract infection. An increasing trend in the resistance of frequently prescribed antibiotics, such as Cephradine, Ampicillin and Co-trimoxazole was observed. The findings of the current study would lead to the rational use of antibiotics for empirical therapy of respiratory tract infections.

\section{REFERENCES}

Bano, S., Tunio, S.A., Mal, S., Jatt, A.N. (2015). Frequency of Methicillin Resistant Staphylococcus aureus Among Isolates of Wound Infections from Hyderabad. Sindh University Research Journal-SURJ (Science Series), 44(4), 683-686.

Bano, S., Tunio, S.A., Memon, A.A., Detho, H., Bano, R., Kumari, K. (2014). Evaluation of Antibiotic Susceptibility Patterns of Uropathogens circulating in Hyderabad, Pakistan. Khyber Medical University Journal, 6(3), 110-115.
El-Mahmood, A.M., Isa, H., Mohammed, A., Tirmidhi, A.B. (2010). Antimicrobial Susceptibility of Some Respiratory Tract Pathogens to Commonly Used Antibiotics at the Specialist Hospital, Yola, Adamawa State, Nigeria. Journal of Clinical Medicine and Research, 2(8), 135-142.

Kunwor, P., Kumaraswamy, M., Siddaraju, M.L., Kafle, B. (2014). Antibiotic Prescribing Pattern in Paediateric Inpatients for Respiratory Tract Infections in Tertiary Care Teaching Hospital. Indo American Journal of Pharmaceutical Research, 4(12), 5630-5635.

Manikandan, C., Amsath, A. (2013). Antibiotic Susceptibility of Bacterial Strains Isolated from Patients with Respiratory Tract Infections. International Journal of Pure And Applied Zoology, 1(1), 61-69.

Rodríguez, M., José, M., Poirel, L., Nordmann, P. (2009). Molecular Epidemiology and Mechanisms of Carbapenem Resistance in Pseudomonas aeruginosa. Antimicrobial Agents and Chemotherapy, 53(11), 4783-4788.

Sharma, S., Agrawal, G. (2016). A Study on Drug Prescribing Pattern in Upper Respiratory Tract Infections Among Children Aged 1-12 Years. International Journal of Basic \& Clinical Pharmacology, 5(2), 406-410.

Siddalingappa, C.M., Kalpana, L., Puli, S., Vasudha, T.K., Acharya, A. (2013). Sensitivity Pattern of Bacteria Causing Respiratory Tract Infections in A Tertiary Care Centre. International Journal of Basic \& Clinical Pharmacology, 2(5), 590-595.

Taura, D.W., Hassan, A., Yayo, A.M., Takalmawa, H. (2013). Bacterial Isolates of The Respiratory Tract Infection and Their Current Sensitivity Pattern Among Patients Attending Aminu Kano Teaching Hospital Kano-Nigeria. Int Res J Microbiol, 4(9), 226-231.

Wayne, P.A. (2010). Clinical \& Laboratory Standard Institute, Performance Standard for Antimicrobial Susceptibility Testing. Twentieth Informational Supplement, 30, M100-M120.

Xirogianni, A., Tsolia, M., Voyiatzi, A., Sioumala, M., Makri, A., Argyropoulou, A., et al. (2013). Diagnosis of Upper and Lower Respiratory Tract Bacterial Infections with The Use of Multiplex PCR Assays. Diagnostics, 3(2), 222-231.

Zafar, A., Hussain, Z., Lomama, E., Sibille, S., Irfan, S. Khan, E. (2008).

Antibiotic Susceptibility of Pathogens Isolated from Patients With

Community-Acquired Respiratory Tract Infections in Pakistan-The Active

Study. Journal of Ayub Medical College, 20(1), 7-9. 